\title{
Vertragsarztsitz
}

\section{Nachbesetzung einer Angestelltenstelle nur bei rechtzeitiger Arbeitsaufnahme}

\author{
Die Nachbesetzung der Stelle eines angestellten Vertragsarztes setzt \\ voraus, dass die Angestelltenstelle durch den vorhergehenden Arzt \\ „real besetzt" wurde. Das hat das Sozialgericht München entschieden.
}

S ofern die erste Anstellungsgenehmigung erloschen ist, fehlt die Grundlage für eine nachfolgende Anstellungsgenehmigung nach $\$ 103$ Abs. 4b S. 3 SGB V urteilte das Sozialgericht (SG) München.

\section{Der Fall}

Die Zulassungsgremien lehnten den Antrag auf Erteilung einer Anstellungsgenehmigung für die Internistin Frau Dr. C. mit einem wöchentlichen Tätigkeitsumfang von 40 Stunden ab. Nachbesetzt werden sollte die Angestelltenstelle eines Arztes, dem zuvor eine Genehmigung erteilt worden war, der die Angestelltentätigkeit jedoch nicht aufgenommen hatte. In dieser wurde bestimmt, dass die $\mathrm{Ge}$ nehmigung endet, sobald die vertragsärztliche Tätigkeit nicht innerhalb von drei Monaten seit Zustellung des Bescheids aufgenommen wird.

Die beim Sozialgericht (SG) München eingelegte Klage richtete sich gegen den Beschluss des Berufungsausschusses, der die Entscheidung des Zulassungsausschusses bestätigte. In einem ebenfalls angefochtenen Widerspruchsbescheid stellte der beklagte Ausschuss fest, dass wegen Nichtaufnahme der Tätigkeit durch Herrn Dr. D. die Genehmigung zu Gunsten des Klägers am 20. Februar 2015 endete. Außerdem sei die Angestelltenstelle nach $\$ 103$ Abs. 4b S. 3 SGB V nicht nachbesetzungsfähig. Aufgrund mehrerer Hinweise ging der Beklagte davon aus, dass der ursprünglich abgebende Arzt von vornherein nicht die Absicht gehabt habe, beim Kläger als angestellter Arzt tätig zu werden. Eine
Nachbesetzung komme nicht in Betracht, da die Voraussetzung hierfür wäre, dass die Angestelltenstelle bereits zuvor durch einen Arzt real besetzt war und ausgeführt wurde.

Gegen diese Entscheidung des Berufungsausschusses wurde ebenfalls Klage beim SG München unter dem Aktenzeichen S 38 KA 1262/15 eingelegt. Das Verfahren wurde in der Sitzung des Gerichts am 29. März 2017 entschieden (Aktenzeichen S 38 KA 1263/15). Der angefochtene Bescheid des beklagten Ausschusses bezüglich der Beendigung der Angestelltengenehmigung von Herrn Dr. D. wurde als rechtmäßig angesehen, weshalb die Klage abgewiesen wurde.

\section{Das Urteil}

Das SG München führt in seiner Begründung des Urteils aus, dass der Widerspruchsbescheid des Beklagten rechtmäßig sei und die Klägerin nicht in ihren Rechten verletzt werde. Denn die Rechtsgrundlage für die Nachbesetzung einer Angestelltenstelle ist $\$ 103 \mathrm{Abs}$. 4b S. 3 SGB V. Die Klägerin hat darauf aber keinen Anspruch. Wie der Beklagte nach der Feststellung des Gerichtes zutreffend ausführt, setzt die grundsätzliche Möglichkeit der Nachbesetzung voraus, dass die Angestelltenstelle durch den vorhergehenden Arzt aktiv besetzt wurde. Die Nachfolgeanstellung ist damit akzessorisch zu der vorhergehenden Anstellung. Ist die erste Anstellungsgenehmigung nach $\$ 103$ Abs. 4b S. 1 SGB V erloschen, weil eine Tätigkeitsaufnahme nicht stattfand, die Tätigkeit nur von geringer Dauer war oder es kei- ne Anhaltspunkte dafür gibt, dass der anzustellende Arzt auf die vertragsärztliche Zulassung verzichtete, um als angestellter Arzt tätig zu werden, fehlt die Grundlage für eine nachfolgende Anstellungsgenehmigung nach $\$ 103 \mathrm{Ab}$ satz 4b S.3 SGB V.

Denn mit dem Wegfall der Erst-Anstellungsgenehmigung entfällt nach Auffassung der Richter auch die Grundlage für die Privilegierung der Anstellungsgenehmigung gegenüber den anderen im Gesetz vorgesehenen Möglichkeiten nach Verzicht auf die vertragsärztliche Zulassung gemäß $\$ 104$ Abs. 4, 3a SGB V. Ansonsten würde dies laut des SG dazu führen, dass die gesetzlichen Regelungen zur Überversorgung umgangen werden könnten und die Anstellungsgenehmigung als Konstrukt letztendlich dem anstellenden Arzt dazu dient, sich einen Vertragsarztsitz zu sichern, um ihn dann kurzfristig anderweitig zu besetzen.

Nachdem das SG in seinem Urteil vom 29. März $2017 \mathrm{zu}$ dem Ergebnis kam, der Beklagte habe zu Recht festgestellt, die Genehmigung zu Gunsten des Klägers ende am 20. Februar 2015 wegen Nichtaufnahme der Tätigkeit durch Herrn Dr. D. und die Angestelltenstelle sei nicht mehr nachbesetzungsfähig, hat die Klägerin keinen Anspruch auf die Nachbesetzung der Angestelltenstelle durch Frau Dr. C.

\section{Literatur beim Verfasser}

\section{Arno Zurstraßen M.A.}

Rechtsanwalt und Mediator im Gesundheitswesen

Fachanwalt für Medizinrecht und Sozialrecht

Aachener Straße 197-199, 50931 Köln

contact@arztundrecht.de

www.arztundrecht.de 